\title{
A!
}

This is an electronic reprint of the original article.

This reprint may differ from the original in pagination and typographic detail.

Deger, Aydin; Brandner, Kay; Flindt, Christian

\section{Lee-Yang zeros and large-deviation statistics of a molecular zipper}

Published in:

Physical Review E

DOI:

10.1103/PhysRevE.97.012115

Published: 12/01/2018

Document Version

Publisher's PDF, also known as Version of record

Please cite the original version:

Deger, A., Brandner, K., \& Flindt, C. (2018). Lee-Yang zeros and large-deviation statistics of a molecular zipper. Physical Review E, 97(1), 1-12. [012115]. https://doi.org/10.1103/PhysRevE.97.012115

This material is protected by copyright and other intellectual property rights, and duplication or sale of all or part of any of the repository collections is not permitted, except that material may be duplicated by you for your research use or educational purposes in electronic or print form. You must obtain permission for any other use. Electronic or print copies may not be offered, whether for sale or otherwise to anyone who is not an authorised user. 


\title{
Lee-Yang zeros and large-deviation statistics of a molecular zipper
}

\author{
Aydin Deger, Kay Brandner, and Christian Flindt \\ Department of Applied Physics, Aalto University, 00076 Aalto, Finland
}

(Received 12 October 2017; published 12 January 2018)

\begin{abstract}
The complex zeros of partition functions were originally investigated by Lee and Yang to explain the behavior of condensing gases. Since then, Lee-Yang zeros have become a powerful tool to describe phase transitions in interacting systems. Today, Lee-Yang zeros are no longer just a theoretical concept; they have been determined in recent experiments. In one approach, the Lee-Yang zeros are extracted from the high cumulants of thermodynamic observables at finite size. Here we employ this method to investigate a phase transition in a molecular zipper. From the energy fluctuations in small zippers, we can predict the temperature at which a phase transition occurs in the thermodynamic limit. Even when the system does not undergo a sharp transition, the Lee-Yang zeros carry important information about the large-deviation statistics and its symmetry properties. Our work suggests an interesting duality between fluctuations in small systems and their phase behavior in the thermodynamic limit. These predictions may be tested in future experiments.
\end{abstract}

DOI: 10.1103/PhysRevE.97.012115

\section{INTRODUCTION}

Continuity is a central concept in physics. Often the properties of a system change only slightly in response to small variations of an external control parameter. Phase transitions, however, are a remarkable exception to this physical principle [1-3]. Consider, for example, a macroscopic gas in contact with a heat reservoir. If the temperature is slowly decreased, the gas may suddenly condense into a liquid and the density of molecules correspondingly increases abruptly. However, how do the molecules "know" that they should suddenly form a liquid rather than a gas? Born and Fuchs first posed this question [4], which for a long time defied a convincing answer.

An early attempt to explain the gas-liquid transition was made by Maxwell and van der Waals [5]. By modifying the ideal gas law to account for the attractive forces between the molecules, they were able to qualitatively explain several experimental observations. However, their theory relied on phenomenological arguments and did not explain how the microscopic configuration of the fluid changes at the condensation point. A more rigorous approach based on statistical mechanics was later proposed by Mayer and co-workers [6-8]. They expanded the free energy of the gas in powers of its density so that the thermodynamic limit could be taken for each term in the expansion. Their cluster expansion provided a quite accurate description of the gas phase. However, it could not correctly account for the properties of the liquid phase.

This drawback of Mayer's method motivated Lee and Yang to develop a nonperturbative approach $[9,10]$. Instead of using an expansion, they analytically continued the partition function into the complex plane by allowing the control parameter to acquire an imaginary part. The zeros of the partition sum in the complex plane of the control parameter are the central objects of this framework. For small volumes, the zeros are well separated from the real axis. As the volume increases, the number of Lee-Yang zeros grows and they move towards the phase transition point on the real axis which they reach in the thermodynamic limit. Since the zeros of the partition function correspond to logarithmic singularities of the free energy, it becomes clear that Mayer's expansion could not converge beyond the critical point. Moreover, one may consider the zeros in the complex plane of any control parameter, e.g., fugacity [9], temperature [11-14], or magnetic field [10], implying that the arguments of Lee and Yang are not restricted
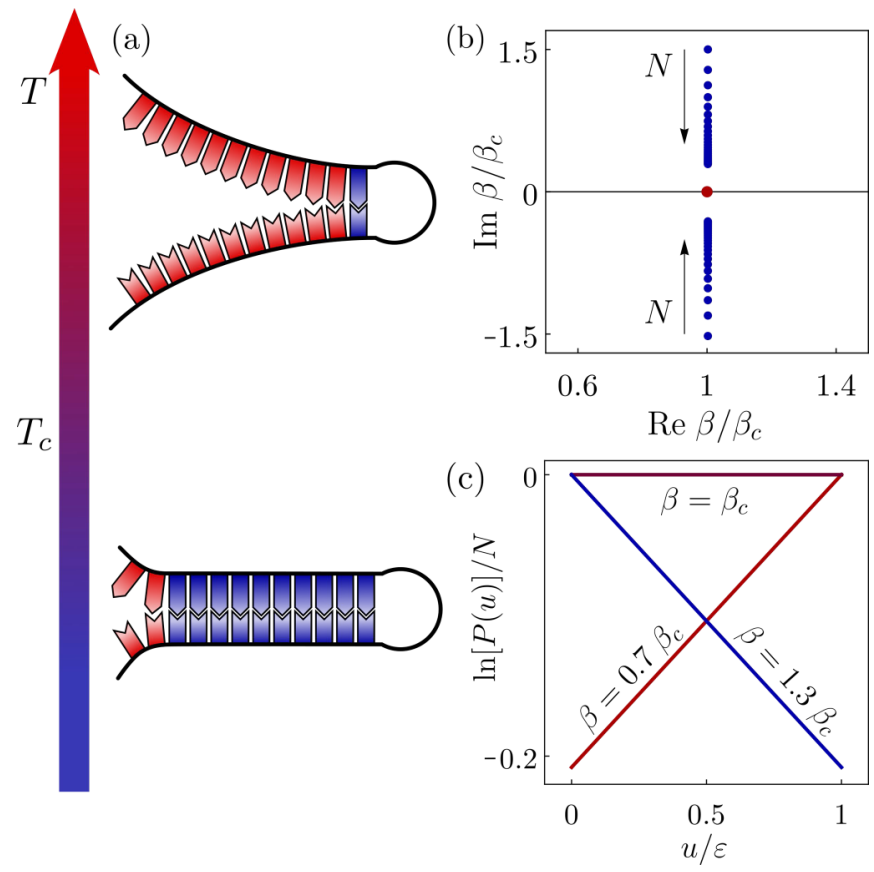

FIG. 1. Zipper model, Lee-Yang zeros, and large-deviation statistics. (a) The molecular zipper is a double-stranded macromolecule held together by $N$ links that can be either open (energy $\varepsilon$ ) or closed (energy 0 ). At the transition temperature $T_{c}$, the system goes through a phase transition. (b) Lee-Yang zeros in the complex plane of the inverse temperature $\beta=1 / k_{B} T$. (c) Large-deviation statistics of the energy per link for large $N$. Distributions are shown for three different temperatures $\beta=0.7 \beta_{c}, \beta_{c}, 1.3 \beta_{c}$, with $\beta_{c}=1 / k_{B} T_{c}$. 
to the gas-liquid transition. Rather, they show that phase transitions generally cannot be described by conventional perturbation methods.

Over the past two decades, the ideas of Lee and Yang have developed into a powerful theoretical tool in statistical physics $[15,16]$ with applications to diverse problems ranging from protein folding $[17,18]$ and percolation $[19,20]$ to complex networks [21,22] and Bose-Einstein condensation [23-26]. Further extensions include the use of Lee-Yang zeros to characterize phase transitions in nonequilibrium systems $[15,16,27]$, quenched quantum systems [28,29], and glass formers [3033]. In addition, a number of experiments have shown that Lee-Yang zeros are not just a theoretical concept; they can also be experimentally determined. In one experiment, the density of Lee-Yang zeros was obtained from magnetization data of a two-dimensional Ising ferromagnet [34]. More recently, LeeYang zeros have been determined by measuring the quantum coherence of a probe spin coupled to an Ising-type spin bath [35,36]. In the context of nonconventional phase transitions [37], Lee-Yang zeros have been determined from real-time measurements of the activity along a quantum-jump trajectory $[38,39]$. For finite-size systems, the Lee-Yang zeros can be extracted from the cumulants of the observable which is conjugate to the control parameter $[38,39]$. With increasing system size, one can then extrapolate the position of the Lee-Yang zeros in the thermodynamic limit and predict a possible phase transition. In the experiment of Ref. [39], the Lee-Yang zeros converged to points that were slightly off from the real axis, corresponding to a crossover rather than a sharp phase transition. Still, the Lee-Yang zeros carried important information about the large-deviation statistics [40], which is typically difficult to measure.

The purpose of this paper is to show that the method developed in Refs. $[38,39]$ is not restricted to the extraction of Lee-Yang zeros from quantum-jump trajectories. The method can in fact be employed to a large class of problems from statistical mechanics. As a specific example, we analyze a simple model [41] of a one-dimensional DNA molecule [42-44] as depicted in Fig. 1(a). The model describes a molecular zipper consisting of a double-stranded macromolecule with infinite-range interactions. We extract the Lee-Yang zeros in the complex plane of the inverse temperature from the energy cumulants of short zippers [Fig. 1(b)]. We can then accurately predict the temperature at which the system undergoes a phase transition in the thermodynamic limit. As we show, the Lee-Yang zeros carry important information about the large-deviation statistics of the energy as illustrated in Fig. 1(c). As an extension, we introduce a finite energy cost for breaking the zipper. In this case, the system exhibits a crossover rather than a sharp phase transition. The Lee-Yang zeros can still be extracted from the energy fluctuations in zippers of finite size and they determine the large-deviation statistics of the energy, as we will see. These findings suggest an interesting duality between fluctuations in small systems and their phase behavior in the thermodynamic limit.

The paper is organized as follows. In Sec. II we introduce the molecular zipper and show that it exhibits a thermal phase transition. In Sec. III we demonstrate how the Lee-Yang zeros in the complex plane of the inverse temperature can be extracted from the energy cumulants for finite-size zippers. In Sec. IV we discuss the connection between the Lee-Yang zeros and the large-deviation statistics. In Sec. V we introduce the broken zipper which exhibits a crossover rather than a sharp phase transition. Still, the Lee-Yang zeros can be extracted from the energy fluctuations. Moreover, in Sec. VI we show that the large-deviation statistics takes the shape of an ellipse whose tilt and width are determined by the Lee-Yang zeros. An associated symmetry of the fluctuations is discussed in Sec. VII. Finally, in Sec. VIII we present our conclusions and provide an outlook on future work. Technical details are deferred to the Appendixes.

\section{ZIPPER MODEL}

Figure 1(a) shows the molecular zipper. The system consists of a double-stranded macromolecule held together by $N$ links that can be either open or closed [41]. If the zipper is fully closed, the internal energy $U$ is zero. The entropy $S$ is also zero, since there is only one configuration for the closed zipper, namely, all links being closed. The free energy at temperature $T$,

$$
F=U-T S,
$$

is then zero for the closed zipper. If $n \leqslant N$ links are open, the energy is $n \varepsilon$, since opening a link requires the energy $\varepsilon>0$. It is only possible to open a link if the preceding one is also open. Hence, links can only open up one after another starting from the floating end. This unwinding mechanism effectively leads to infinite-range interactions between distant links. Each link can open in $g$ different ways such that $n$ links can be open in $g^{n}$ different configurations with the corresponding entropy $S=$ $k_{B} \ln g^{n}$, where $k_{B}$ is Boltzmann's constant. The free energy then becomes

$$
F=n\left(\varepsilon-k_{B} T \ln g\right), \quad 0 \leqslant n \leqslant N .
$$

The free energy per link can be expressed as

$$
\frac{F}{N \varepsilon}=\frac{n}{N}\left(1-T / T_{c}\right)
$$

where we have defined the temperature

$$
T_{c}=\frac{\varepsilon}{k_{B} \ln g} .
$$

The free energy per link corresponding to different numbers of open links is shown in Fig. 2(a).

In equilibrium, the system minimizes the free energy [1]. Thus, at low temperatures, where the free energy is dominated by the internal energy, the lowest free energy is zero, corresponding to the closed zipper. With increasing temperature, the free energy becomes dominated by the entropy such that the free energy is now maximally negative for the fully open zipper. The transition between the two phases occurs at the temperature $T_{c}$, where the free energies are equal. The equilibrium free energy is also shown in Fig. 2(a). We clearly see that the equilibrium free energy is nonanalytic at $T=T_{c}$, where the free energy difference between the two phases vanishes. We note that there is no phase transition for $g=1$, where the temperature $T_{c}$ diverges.

The discussion above is based on simple thermodynamic arguments. A more rigorous analysis requires that we calculate 

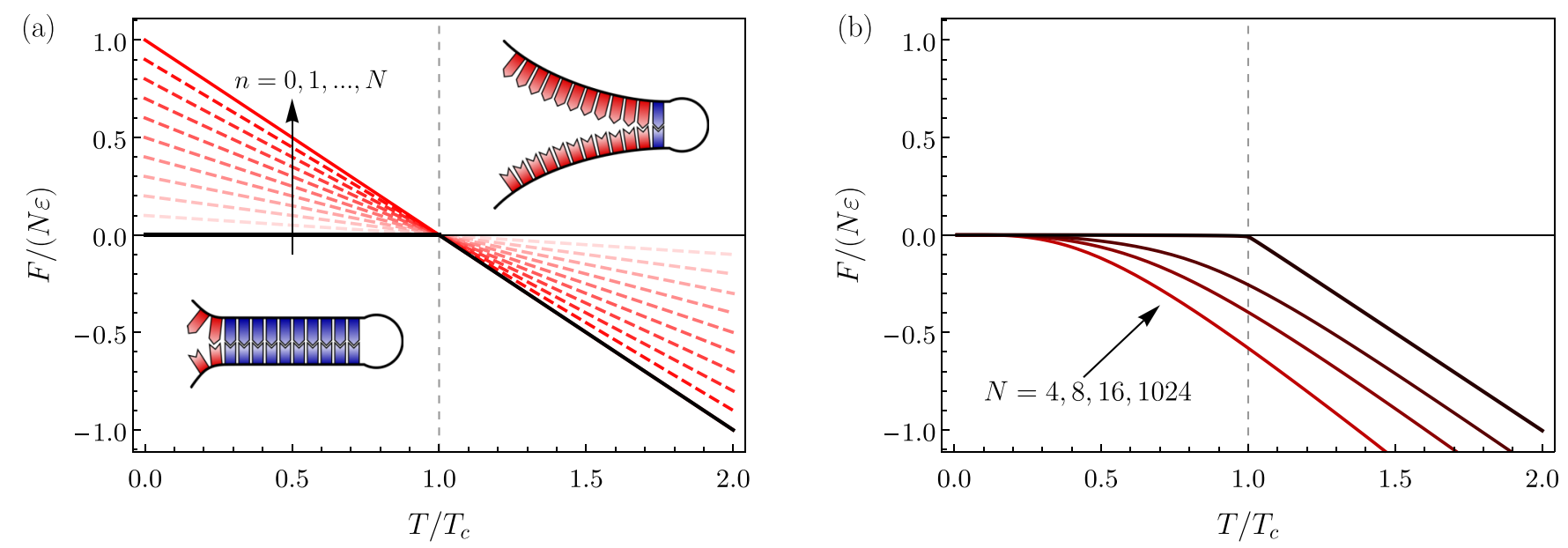

FIG. 2. Free energy of the molecular zipper. (a) Free energy per link as a function of the temperature $T$ for different numbers of open links $n=0,1, \ldots, N$. For $T<T_{c}$, the closed zipper has the lowest free energy. For $T>T_{c}$, the open zipper has the lowest free energy. The equilibrium free energy is shown with a thick black line. (b) Equilibrium free energy per link for different lengths of the zipper. In the thermodynamic limit, the equilibrium free energy per link becomes nonanalytic at $T=T_{c}$.

the canonical partition function. For the zipper model, the partition function can easily be expressed as a sum over all possible numbers of open links

$$
Z=\sum_{n=0}^{N} g^{n} e^{-\beta n \varepsilon}=\frac{1-\left(g e^{-\beta \varepsilon}\right)^{N+1}}{1-g e^{-\beta \varepsilon}},
$$

having introduced the inverse temperature $\beta=1 / k_{B} T$. The free energy for finite $N$ can then be obtained as

$$
F=-\beta^{-1} \ln Z=-\beta^{-1} \ln \left[\frac{1-\left(g e^{-\beta \varepsilon}\right)^{N+1}}{1-g e^{-\beta \varepsilon}}\right] .
$$

In Fig. 2(b), we show the equilibrium free energy as a function of the temperature. With increasing length, the free energy per link approaches the thermodynamic value

$$
\frac{F}{N \varepsilon}= \begin{cases}1-T / T_{c}, & T>T_{c} \\ 0, & T \leqslant T_{c} .\end{cases}
$$

The two cases correspond exactly to the free energies of the open and the closed zipper, above and below the critical temperature, respectively. Interestingly, the zipper has to be rather long $N \sim 10^{3}$ before it becomes apparent that the free energy will become nonanalytic at $T_{c}$.

From the free energy, we find the average energy as $\langle U\rangle=$ $\partial_{\beta}(\beta F)$. The average energy per link as a function of the temperature is shown in Fig. 3(a). Again, with increasing length, the average energy per link approaches the thermodynamic value

$$
\frac{\langle U\rangle}{N \varepsilon}= \begin{cases}1, & T>T_{c} \\ 1 / 2, & T=T_{c} \\ 0, & T<T_{c}\end{cases}
$$

This result shows that the system exhibits a first-order phase transition at $T=T_{c}$, where the average energy per link jumps abruptly from 0 to $\varepsilon$.

From the free energy, we also obtain the variance of the energy fluctuations as $\left\langle\left\langle U^{2}\right\rangle\right\rangle=-\partial_{\beta}^{2}(\beta F)$. In Fig. 3(b), we show the variance per link as a function of the temperature.
Again, with increasing length, the variance approaches the thermodynamic value

$$
\frac{\left\langle\left\langle U^{2}\right\rangle\right\rangle}{N \varepsilon^{2}}= \begin{cases}0, & T \neq T_{c} \\ \frac{N}{12}, & T=T_{c}\end{cases}
$$

which diverges with the system size $N$ exactly at the critical temperature. Also for the average energy and the variance, the zipper has to be rather long before the phase transition becomes apparent.

\section{LEE-YANG ZEROS AND HIGH CUMULANTS}

To understand how the free energy can become nonanalytic in the thermodynamic limit, Lee and Yang suggested to investigate the zeros of the partition function in the complex plane of the control parameters $[9,10]$. In the case of the zipper model, we consider the zeros of the partition function for complex values of the inverse temperature $\beta$. (Zeros in the complex plane of the inverse temperature are sometimes referred to as Fisher zeros [11].) For real values of $\beta$, it is clear that the partition function in Eq. (5) cannot vanish, since it is a sum of positive terms. On the other hand, treating $\beta$ as a complex variable, the partition sum vanishes at the points

$$
\beta_{k}=\beta_{c}+\frac{2 \pi k}{\varepsilon(N+1)} i,
$$

where $\beta_{c}=1 / k_{B} T_{c}$ is the inverse transition temperature and $k \in\{-N, \ldots, N\} \backslash\{0\}$ if we restrict ourselves to the stripe $-\pi \leqslant \operatorname{Im} \beta \varepsilon \leqslant \pi$ in the complex plane. In Fig. 4(a), we show the motion of the Lee-Yang zeros that are closest to the real axis with increasing system size. As expected from Eq. (10), the Lee-Yang zeros approach the inverse phase transition temperature $\beta_{c}$ in the thermodynamic limit. We note that the perpendicular approach to the real axis signals that the system is exhibiting a first-order phase transition [12,14,15]. To illustrate how one may pinpoint the exact convergence point from finite-size systems, we show in Fig. 4(b) the real part and the imaginary part of the leading Lee-Yang zeros as functions 

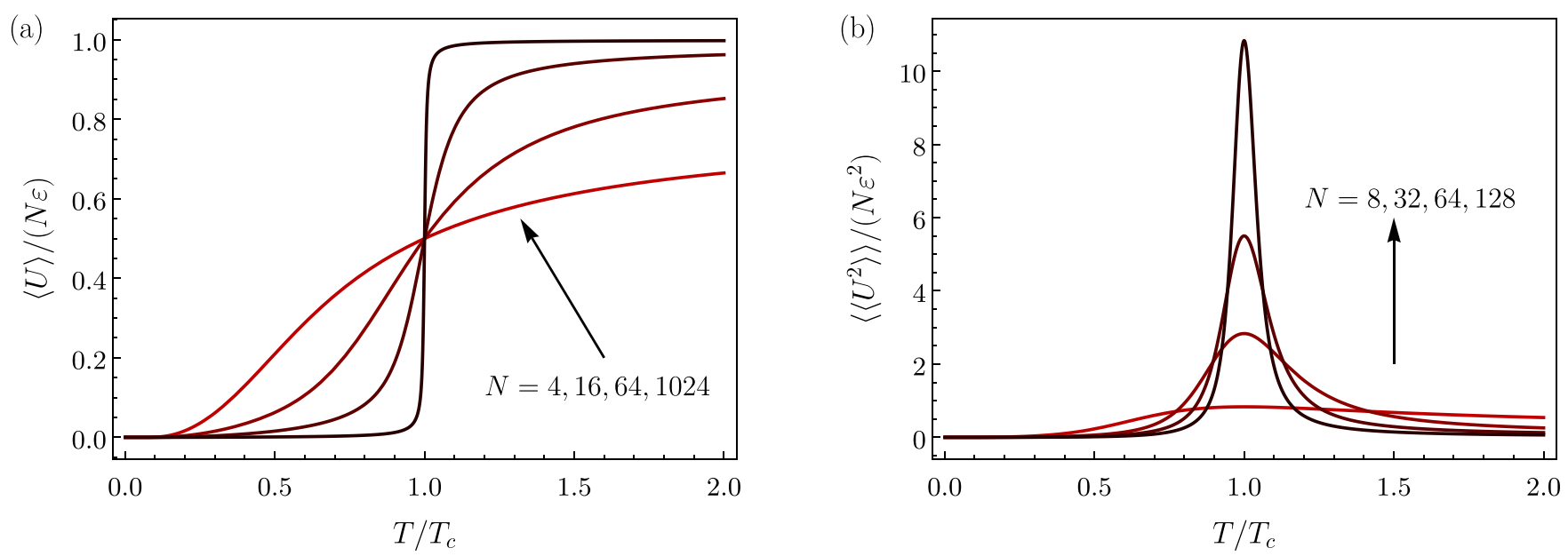

FIG. 3. Average energy and fluctuations. (a) Average energy per link as a function of the temperature $T$. The various curves correspond to different lengths of the zipper. In the thermodynamic limit, the average energy per link develops a discontinuity at the transition temperature $T_{c}$. (b) Variance of the energy for zippers of different lengths. The energy fluctuations diverge [45] at the transition temperature $T_{c}$ in the thermodynamic limit.

of the inverse system size. From such a figure, one can easily extrapolate the position of the Lee-Yang zeros in the large system-size limit.

Since the partition sum at finite size is an entire function, we can write it in terms of the Lee-Yang zeros as [46]

$$
Z(\beta)=Z(0) e^{\beta c} \prod_{k}\left(1-\beta / \beta_{k}\right),
$$

where the constant $c=Z^{\prime}(0) / Z(0)+\sum_{k} 1 / \beta_{k}$ is independent of $\beta$. In general, it is challenging to determine the LeeYang zeros of a partition function and perform the product expansion. However, due to the simplicity of the zipper model, we can find all Lee-Yang zeros and explicitly construct the expansion as shown in Appendix A.

The free energy can now be written as

$$
F(\beta)=-\beta^{-1}\left[\ln Z(0)+\beta c+\sum_{k} \ln \left(1-\beta / \beta_{k}\right)\right] .
$$

This expression shows that the phase behavior of the system is fully encoded in the Lee-Yang zeros. We also see that the Lee-Yang zeros correspond to logarithmic singularities of the free energy. Moreover, as $N$ increases, the number of zeros grows and they approach the point $\beta=\beta_{c}$ on the real axis, where $F(\beta)$ becomes nonanalytic. We note that the partition function in Eq. (5) does not vanish at $\beta_{c}$ in the limit $N \rightarrow \infty$. Rather, it becomes a discontinuous function along the vertical line in the complex plane that crosses the real axis at $\beta=\beta_{c}$.

Lee-Yang zeros are useful to understand phase transitions. However, until recently, it has been unclear how to determine Lee-Yang zeros in experiment. As we will now discuss, the Lee-Yang zeros can be obtained from the fluctuations of thermodynamic observables of finite-size systems $[38,39,47,48]$. To this end, we first note that the partition function delivers the moments of the energy upon differentiation with respect to the inverse temperature as

$$
\left\langle U^{n}\right\rangle=\left[\left(-\partial_{\beta}\right)^{n} Z\right] / Z .
$$

Similarly, the cumulants can be obtained as

$$
\left\langle\left\langle U^{n}\right\rangle\right\rangle=\left(-\partial_{\beta}\right)^{n} \ln Z=\left(-\partial_{\beta}\right)^{n}(-\beta F) .
$$

Now, using Eq. (12), we can express the cumulants as

$$
\left\langle\left\langle U^{n}\right\rangle\right\rangle=(-1)^{n-1} \sum_{k} \frac{(n-1) !}{\left(\beta_{k}-\beta\right)^{n}}, \quad n>1 .
$$

The fact that the Lee-Yang zeros come in complex-conjugate pairs ensures that the cumulants are real.

The result above shows that the energy fluctuations are fully described by the Lee-Yang zeros. Moreover, we see that the high cumulants essentially are governed by the pair of LeeYang zeros that are closest to the inverse temperature $\beta$ on the real axis, since they dominate the sum for high orders $n \gg 1$. The contributions from subleading zeros are suppressed with the distance to $\beta$ and the order $n$. Now writing the closest pair of Lee-Yang zeros $\beta_{\mathrm{o}}$ and $\beta_{\mathrm{o}}^{*}$ in polar coordinates as [see Fig. 4(a)]

$$
\beta_{\mathrm{o}}=\rho_{\mathrm{o}} e^{i \phi_{\mathrm{o}}}+\beta,
$$

we can approximate the sum as

$$
\left\langle\left\langle U^{n}\right\rangle\right\rangle(-1)^{n-1}(n-1) ! \frac{2 \cos \left(n \phi_{\mathrm{o}}\right)}{\rho_{\mathrm{o}}^{n}}, n \gg 1 .
$$

The approximation becomes better with increasing order and it shows how the high cumulants are determined by the closest Lee-Yang zeros. We also see that the high cumulants should oscillate, for example, as a function of the order $n$ or the inverse temperature $\beta$. Such oscillations are generic features of high derivatives [49-51] and they have been observed in measurements of the high cumulants of the electron counting statistics in quantum dots [52-55].

Equation (17) is important as it relates the Lee-Yang zeros to experimental observables. In particular, by measuring the high cumulants of the energy, one can extract the position of the leading pair of Lee-Yang zeros. To this end, we solve Eq. (17) 

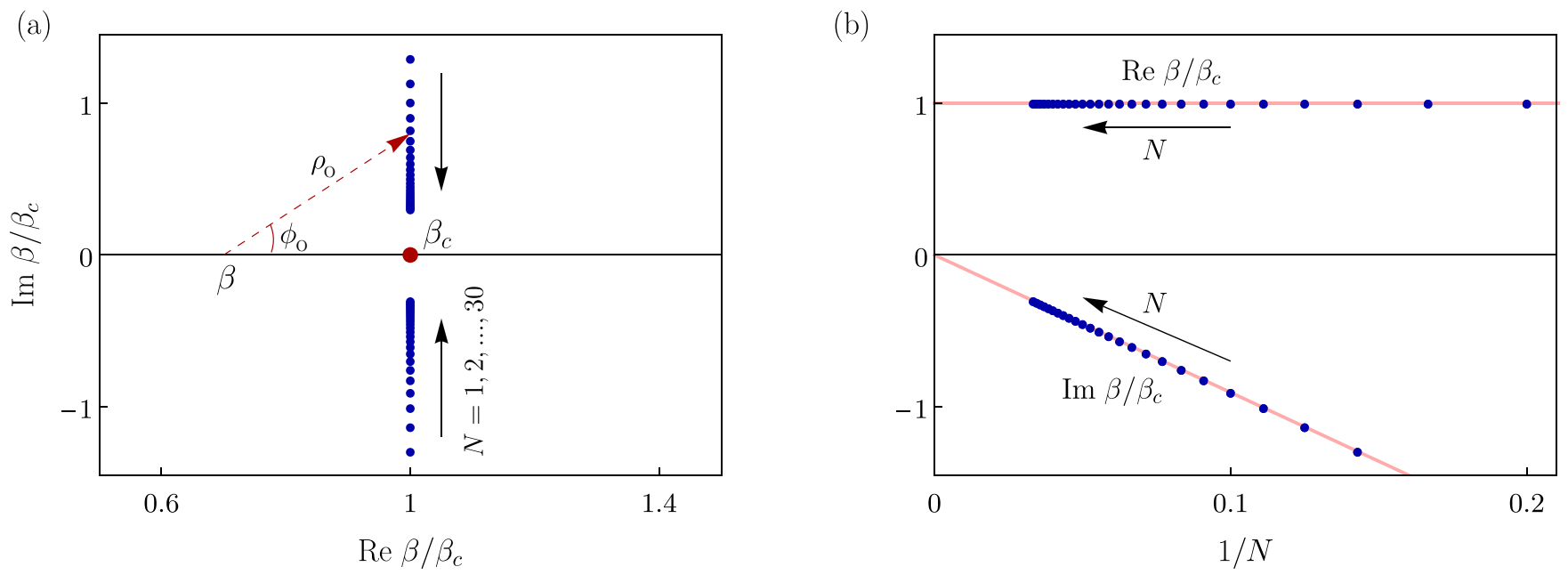

FIG. 4. Lee-Yang zeros for the zipper model. (a) Exact results for the leading Lee-Yang zeros in the complex plane of the inverse temperature for zippers of increasing size $N=1,2, \ldots, 30$. In the thermodynamic limit, the Lee-Yang zeros reach the inverse temperature on the real axis for which a phase transition occurs. The polar coordinates $\rho_{\mathrm{o}}$ and $\phi_{\mathrm{o}}$ defined in Eq. (16) are indicated. (b) Extrapolation of the real part and the imaginary part of the Lee-Yang zeros in the thermodynamic limit.

for $\rho_{\mathrm{o}}$ and $\phi_{\mathrm{o}}$ and find $[38,51,55,56]$

$$
\left[\begin{array}{cc}
1 & \frac{-\kappa_{n}^{(+)}}{n} \\
1 & \frac{-\kappa_{n+1}^{(+)}}{n+1}
\end{array}\right]\left[\begin{array}{c}
-2 \rho_{\mathrm{o}} \cos \phi_{\mathrm{o}} \\
\rho_{\mathrm{o}}^{2}
\end{array}\right]=\left[\begin{array}{c}
(n-1) \kappa_{n}^{(-)} \\
n \kappa_{n+1}^{(-)}
\end{array}\right]
$$

in terms of the ratios of cumulants

$$
\kappa_{n}^{( \pm)} \equiv\left\langle\left\langle U^{n \pm 1}\right\rangle\right\rangle /\left\langle\left\langle U^{n}\right\rangle\right\rangle
$$

Thus, from four consecutive cumulants, one may extract the leading pair of Lee-Yang zeros by solving Eq. (18).

To illustrate the method, we extract the leading Lee-Yang zeros for the zipper model using the cumulants of the energy. In Fig. 5(a), we show the motion of the leading Lee-Yang zeros in the complex plane of the inverse temperature. With increasing system size, the Lee-Yang zeros move towards the real axis. In Fig. 5(b), we again show the real part and the imaginary part of the Lee-Yang zeros as functions of the inverse system size, allowing us to extract the exact convergence point. We see that the accuracy of the method improves with the order of the energy cumulants. We note that the method is not restricted to energy cumulants and Lee-Yang zeros in the complex plane of the inverse temperature. Other sets of conjugate variables would also work.

\section{LARGE-DEVIATION STATISTICS}

Having illustrated our method for determining the Lee-Yang zeros from experimental observables, we now go on to discuss the implications for the large-deviation statistics of the energy [40]. To this end, we evaluate the probability $P(u)$ for finding the zipper with the energy $u=n \varepsilon / N$ per link. We can express this probability as

$$
P(u)=\frac{g^{n} e^{-\beta N u}}{Z}
$$

with the partition function $Z$ given by Eq. (5). We focus on the tails of the distribution by considering the logarithm of the probability distribution

$$
\frac{\ln P(u)}{N}=u\left(\beta_{c}-\beta\right)+\frac{\beta F}{N} .
$$

For long zippers $N \gg 1$, we can use Eq. (7) for the free energy and we then find the simple expression

$$
\frac{\ln P(u)}{N}= \begin{cases}\left(\beta_{c}-\beta\right) u, & \beta \geqslant \beta_{c} \\ \left(\beta_{c}-\beta\right)(u-\varepsilon), & \beta<\beta_{c},\end{cases}
$$

which vanishes exactly at the phase transition $\beta=\beta_{c}$. In Fig. 1(c), we show the large-deviation function for temperatures below, equal to, or above the phase transition temperature. The large-deviation function describes the exponential decay of the probability to observe energy fluctuations away from the average value. Equation (22) shows that the large-deviation function is determined by the inverse transition temperature $\beta_{c}$. As we will see below, this behavior is of a more general nature, namely, the convergence points of the Lee-Yang zeros determine the large-deviation statistics of thermodynamic observables.

Before closing this section, we briefly discuss the cumulants of the energy in the thermodynamic limit. Away from the phase transition, the free energy and all cumulants of the energy grow linearly with the system size $N$. On the other hand, at the phase transition we find

$$
\left\langle\left\langle U^{n}\right\rangle\right\rangle=\varepsilon^{n} \zeta(1-n)\left[1-(N+1)^{n}\right],
$$

where $\zeta(x)$ is the Riemann zeta function, which for the first few cumulants takes the values $\zeta(0)=-1 / 2, \zeta(-1)=-1 / 12$, $\zeta(-2)=0$, and $\zeta(-3)=-1 / 120$. This particular behavior of the cumulants is closely related to the strongly non-Gaussian energy fluctuations at the phase transition. Specifically, if we introduce the variable

$$
\vartheta=\frac{U-\langle U\rangle}{\sqrt{\left\langle\left\langle U^{2}\right\rangle\right\rangle}}
$$



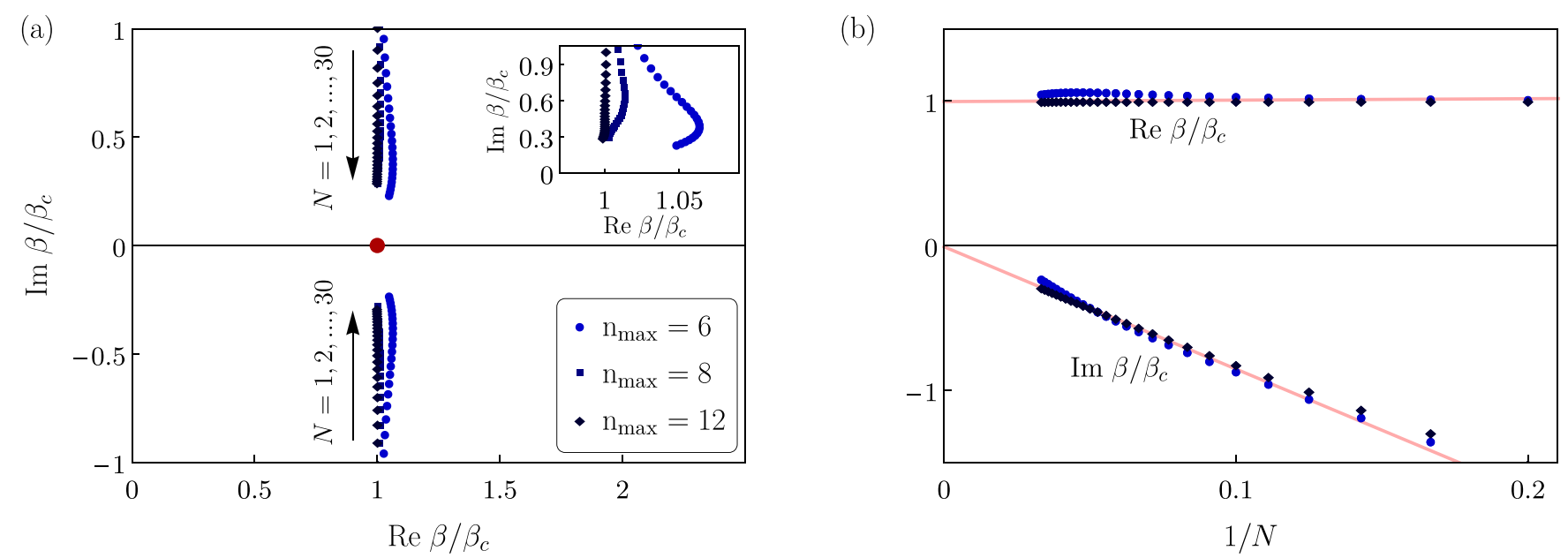

FIG. 5. Extraction of Lee-Yang zeros from the energy cumulants. (a) The Lee-Yang zeros are extracted from four consecutive cumulants up to order $n_{\max }$ for zippers of length $N=1,2, \ldots, 30$. The inverse temperature is $\beta=0.75 \beta_{c}$. The inset illustrates how the accuracy of the method is improved by increasing the cumulant order. (b) Extrapolation of the real and imaginary parts of the Lee-Yang zeros in the thermodynamic limit. These results should be compared with the exact ones in Fig. 4.

which measures deviations from the average energy in units given by the variance [57], we find $\langle\vartheta\rangle=0,\left\langle\left\langle\vartheta^{2}\right\rangle\right\rangle=1$, and $\left\langle\left\langle\vartheta^{n}\right\rangle\right\rangle \simeq 0$ for $n>2$, away from the phase transition. This shows that $\vartheta$ essentially is governed by a Gaussian distribution with only small deviations in the tails. By contrast, at the phase transition, we find

$$
\left.\left\langle\vartheta^{n}\right\rangle\right\rangle=-\zeta(1-n)(2 \sqrt{3})^{n}, \quad n \geqslant 2
$$

in the thermodynamic limit. Thus, the scaled high-order cumulants are nonzero, reflecting the highly non-Gaussian fluctuation of $\vartheta$ at the phase transition.

\section{BROKEN ZIPPER}

We are now ready to illustrate our method with a more evolved model. To this end, we consider finite values of the energy cost $J$ associated with opening a link inside a closed part of the zipper. In this case, the total energy of the system can be expressed as [58]

$$
U=\varepsilon \sum_{i=1}^{N} \sigma_{i}+J \sum_{i=2}^{N} \sigma_{i}\left(1-\sigma_{i-1}\right),
$$

where $\sigma_{i}=1$ for open links and $\sigma_{i}=0$ for closed links. The corresponding partition function reads

$$
Z=\sum_{\left\{\sigma_{i}\right\}} g\left(\left\{\sigma_{i}\right\}\right) e^{-\beta U\left(\left\{\sigma_{i}\right\}\right)},
$$

where the degeneracy factor associated with a particular sequence $\left\{\sigma_{i}\right\}$ is

$$
g\left(\left\{\sigma_{i}\right\}\right)=g^{\sum_{i}^{N} \sigma_{i}}
$$

recalling that each link can be open in $g>1$ ways. The partition function can now be written as

$$
Z=\sum_{\left\{\sigma_{i}\right\}} g^{\sigma_{1}} e^{-\beta \varepsilon \sigma_{1}} \prod_{i=2}^{N} T\left(\sigma_{i}, \sigma_{i-1}\right),
$$

where

$$
T\left(\sigma_{i}, \sigma_{i-1}\right)=g^{\sigma_{i}} e^{-\beta(J+\varepsilon) \sigma_{i}} e^{\beta J \sigma_{i} \sigma_{i-1}}
$$

are elements of the transfer matrix

$$
\mathbf{T}=\left(\begin{array}{cc}
g e^{-\beta \varepsilon} & g e^{-\beta(J+\varepsilon)} \\
1 & 1
\end{array}\right) .
$$

Imposing the appropriate boundary conditions, the partition sum can be expressed in the compact form

$$
Z=\left\langle-\left|\mathbf{T}^{N}\right| 1\right\rangle,
$$

with $\langle-|=(1,1)$ and $|1\rangle=(1,0)^{T}$. This expression allows us to calculate the partition function and the free energy for zippers of finite size together with the cumulants of the energy. The partition function in Eq. (5) is readily recovered in the limit $J \rightarrow \infty$. From Eq. (32) we also see that the partition sum must take the form

$$
Z=c_{1} \lambda_{1}^{N}+c_{2} \lambda_{2}^{N},
$$

where $\lambda_{1}$ and $\lambda_{2}$ are the eigenvalues of the transfer matrix and the prefactors $c_{1}$ and $c_{2}$ are independent of the system size. In the thermodynamic limit, the free energy

$$
\frac{F}{N}=-k_{B} T \ln \lambda_{\max }
$$

is thus given by the eigenvalue of the transfer matrix with the largest absolute value. Correspondingly, we see that nonanalyticities in the free energy, associated with phase transitions, are given by eigenvalue crossings of the transfer matrix, i.e., values of $\beta$ for which $\lambda_{1}=\lambda_{2}$. We also see that the Lee-Yang zeros with increasing system size approach these values of $\beta$. Specifically, by solving for the zeros of the partition function, we find

$$
\ln \left(\lambda_{1}\right)=\ln \left(\lambda_{2}\right)+\frac{\ln \left(c_{2} / c_{1}\right)+i \pi(2 k+1)}{N}
$$

for integer $k$. Thus, the Lee-Yang zeros will move towards the values of $\beta$ for which $\lambda_{1}=\lambda_{2}$, since the second term on the right-hand side vanishes for $N \rightarrow \infty$. This conclusion holds 

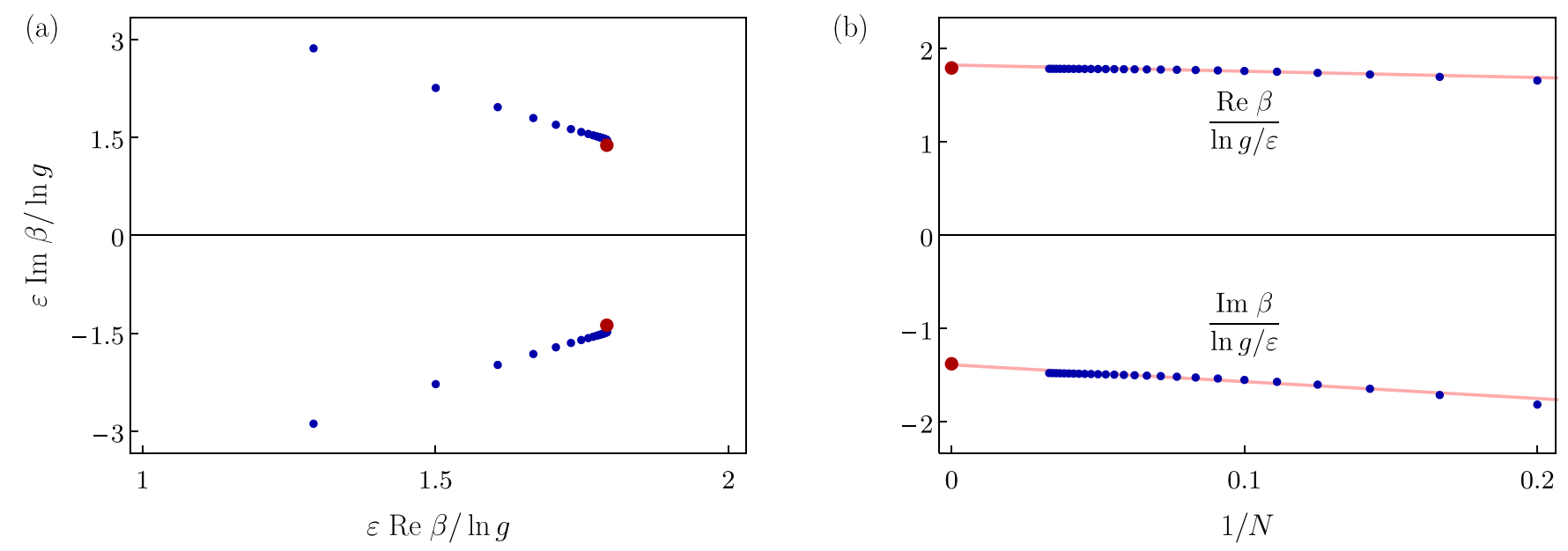

FIG. 6. Lee-Yang zeros for the broken zipper. (a) Leading Lee-Yang zeros in the complex plane of the inverse temperature. The zeros are extracted from the energy cumulants $\left\langle\left\langle U^{n}\right\rangle\right\rangle$ of order $n=9,10,11,12$ for zippers of length $N=1,2, \ldots, 30$ with $J=\varepsilon$ and $\beta=1.75(\ln g) / \varepsilon$. With increasing size, the Lee-Yang zeros move towards the convergence points marked with big red circles. (b) Extrapolation of the convergence points $\beta_{c}$ and $\beta_{c}^{*}$ in the thermodynamic limit. The zeros remain complex.

for transfer matrices of any size, where the phase transition is determined by a crossing of the two largest eigenvalues.

The two eigenvalues of the transfer matrix are

$$
\lambda_{1,2}=\frac{1+g e^{-\beta \varepsilon}}{2} \pm \sqrt{\left(\frac{1-g e^{-\beta \varepsilon}}{2}\right)^{2}+g e^{-\beta(\varepsilon+J)}} .
$$

An eigenvalue crossing occurs when the argument of the square root is zero. For real values of $\beta$, this can only happen with an infinitely large $J$. In that case, the system exhibits a phase transition for $\beta=(\ln g) / \varepsilon$ as we saw earlier on. For finite values of $J$, eigenvalue crossings can only occur for complex values of $\beta$ and the system does not exhibit a sharp phase transition at any temperature.

We find the Lee-Yang zeros from the high cumulants of the energy using the extraction scheme in Eq. (18). An example of this procedure is shown in Fig. 6(a). The dominating LeeYang zeros are extracted for short zippers of a finite length. To find the convergence points of the Lee-Yang zeros, we need to extrapolate their position in the thermodynamic limit. To this end, we note that $1 / N$ appears as a natural expansion parameter, e.g., in Eq. (35). We therefore expand the Lee-Yang zeros as

$$
\begin{aligned}
& \operatorname{Re}\left[\beta_{\mathrm{o}}\right]=\operatorname{Re}\left[\beta_{c}\right]+\alpha_{1}^{\mathrm{Re}} / N+\alpha_{2}^{\mathrm{Re}} / N^{2}+\cdots, \\
& \operatorname{Im}\left[\beta_{\mathrm{o}}\right]=\operatorname{Im}\left[\beta_{c}\right]+\alpha_{1}^{\operatorname{Im}} / N+\alpha_{2}^{\operatorname{Im}} / N^{2}+\cdots,
\end{aligned}
$$

with unknown expansion parameters. With an infinitely large $J$, we can indeed write the dominating Lee-Yang zeros from Eq. (10) as $\operatorname{Re}\left[\beta_{\mathrm{o}}\right]=\operatorname{Re}\left[\beta_{c}\right]$ and $\operatorname{Im}\left[\beta_{\mathrm{o}}\right]= \pm(2 \pi / \varepsilon)(1 / N-$ $\left.1 / N^{2}+1 / N^{3}+\cdots\right)$. For finite values of $J$ we assume that such a system-size dependence still holds. We can then use linear interpolation in $1 / N$ with increasing system size. In Fig. 6(b) we show the extraction of the convergence points in the thermodynamic limit. We see that the Lee-Yang zeros in this case remain complex, corresponding to a crossover rather than a sharp phase transition. However, as we will now see, the Lee-Yang zeros still carry important information about the large-deviation statistics and its symmetry properties.

\section{LARGE DEVIATIONS AND LEE-YANG ZEROS}

We can now connect the Lee-Yang zeros with the largedeviation statistics of the energy fluctuations [40,59]. To this end, we first write the partition sum as

$$
Z=\sum_{n=0}^{N} \sum_{m=0}^{N-n} g^{n}\left(\begin{array}{l}
n \\
m
\end{array}\right)\left(\begin{array}{c}
N-n \\
m
\end{array}\right) e^{-\beta(n \varepsilon+m J)}
$$

Here the energy and degeneracy factor associated with having $n$ open links are $n \varepsilon$ and $g^{n}$, respectively, and $m J$ is the energy associated with having $m$ open segments inside the zipper. The corresponding degeneracy factor is given by the product of binomial coefficients which follows from simple combinatorial arguments.

The probability density of finding the zipper with energy $u=U / N$ per link can be written as

$$
P(u)=N \sum_{n=0}^{N} \sum_{m=0}^{N-n} \frac{g^{n}\left(\begin{array}{c}
n \\
m
\end{array}\right)\left(\begin{array}{c}
N-n \\
m
\end{array}\right) e^{-\beta N u}}{Z} \delta(n \varepsilon+m J-N u) .
$$

This expression forms the basis of our exact calculations of the large-deviation statistics of the energy fluctuations. However, to connect the large-deviation statistics to the Lee-Yang zeros, we proceed with an approximation for large systems. Specifically, for $N \gg 1$, we can approximate the probability density as

$$
P(u) \simeq \frac{N}{2 \pi} \int_{-\infty}^{+\infty} d \gamma \frac{Z(\beta+i \gamma)}{Z(\beta)} e^{i \gamma N u},
$$

as shown in Appendix B. For large systems, the partition sum is determined by the largest eigenvalue of the transfer matrix such that

$$
P(u) \simeq \frac{N}{2 \pi} \int_{-\infty}^{+\infty} d \gamma\left[\frac{\lambda_{\max }(\beta+i \gamma)}{\lambda_{\max }(\beta)}\right]^{N} e^{i \gamma N u} .
$$



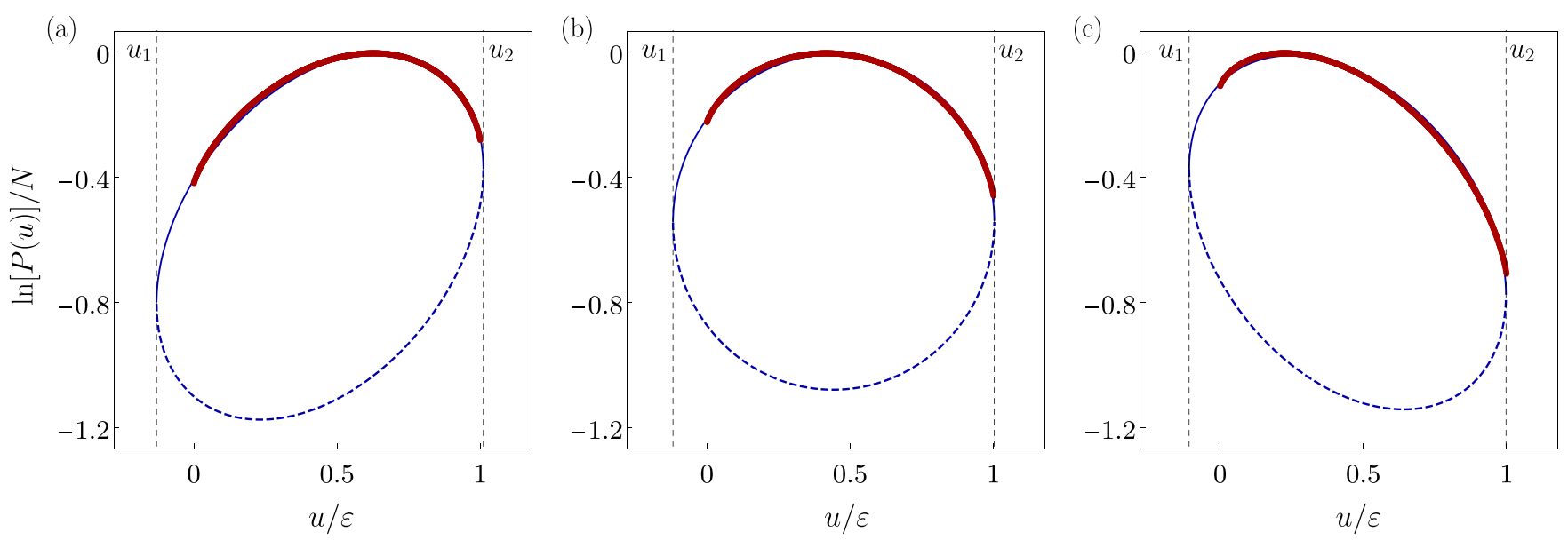

FIG. 7. Large-deviation statistics for the broken zipper. The inverse temperature is (a) $\beta=0.7 \operatorname{Re}\left[\beta_{\mathrm{c}}\right]$, (b) $\beta=\operatorname{Re}\left[\beta_{\mathrm{c}}\right]$, and (c) $\beta=$ $1.3 \operatorname{Re}\left[\beta_{\mathrm{c}}\right]$, where $\operatorname{Re}\left[\beta_{\mathrm{c}}\right]$ is the real part of the convergence points extracted in Fig. 6 with $J=\varepsilon$. The thick red lines are exact results based on Eq. (39) with $N=10^{3}$, while the blue lines are the top of the ellipse described by Eq. (48). The fitting parameters $u_{1}, u_{2}$, and $\mathcal{N}$ depend weakly on the temperature. The lower parts of the ellipses are indicated with dashed lines.

Now, via a change of variable, we obtain the expression

$P(u) \simeq \frac{N}{2 \pi i} \int_{\beta-i \infty}^{\beta+i \infty} d \gamma \exp \left\{N\left[(\gamma-\beta) u+\ln \left(\frac{\lambda_{\max }(\gamma)}{\lambda_{\max }(\beta)}\right)\right]\right\}$,

which is suitable for a saddle-point approximation in the large$N$ limit. To find the saddle points, we need to examine the exponent of the integrand and we thus define

$$
\Theta(\gamma)=(\gamma-\beta) u+\ln \left(\frac{\lambda_{\max }(\gamma)}{\lambda_{\max }(\beta)}\right) .
$$

The saddle points are given by values of $\gamma$ for which the first derivative of $\Theta(\gamma)$ vanishes, i.e., $\Theta^{\prime}\left(\gamma_{0}\right)=0$. To find the saddle points, we note that the integral should be dominated by the square-root branch points of $\lambda_{\max }(\gamma)$ closest to the real axis. Hence, we make the ansatz

$$
\Theta(\gamma) \simeq(\gamma-\beta) u+c_{1}+c_{2} \gamma \pm c_{3} \sqrt{\left(\beta_{c}-\gamma\right)\left(\beta_{c}^{*}-\gamma\right)}
$$

with plus for $\operatorname{Re}[\gamma]<\operatorname{Re}\left[\beta_{c}\right]$ and minus for $\operatorname{Re}[\gamma]>\operatorname{Re}\left[\beta_{c}\right]$. Here $\beta_{c}$ and $\beta_{c}^{*}$ are the convergence points of the Lee-Yang zeros in the thermodynamic limit and $c_{1}, c_{2}$, and $c_{3}$ are unknown constants. This ansatz captures the analytic structure of $\Theta(\gamma)$ around $\beta_{c}$ and $\beta_{c}^{*}$.

The first derivative of $\Theta(\gamma)$ now becomes

$$
\Theta^{\prime}(\gamma)=u+c_{2} \pm c_{3} \frac{\gamma-\operatorname{Re}\left[\beta_{c}\right]}{\sqrt{\left(\gamma-\operatorname{Re}\left[\beta_{c}\right]\right)^{2}+\operatorname{Im}\left[\beta_{c}\right]^{2}}} .
$$

For the saddle points we then find

$$
\gamma_{0}(u)=\operatorname{Re}\left[\beta_{c}\right] \pm \operatorname{Im}\left[\beta_{c}\right] \frac{\left|u+c_{2}\right|}{\sqrt{c_{3}^{2}-\left(u+c_{2}\right)^{2}}},
$$

where the sign should be chosen consistently with Eq. (44). Inserting this result back into the ansatz and defining
$\mathcal{M}=c_{1}+c_{2} \operatorname{Re}\left[\beta_{c}\right]$ and $u_{1,2}=-c_{2} \pm c_{3}$, we find, for the large-deviation function $\ln [P(u)] / N \simeq \Theta\left(\gamma_{0}\right)$,

$\frac{\ln P(u)}{N} \simeq \mathcal{M}+\left(\operatorname{Re}\left[\beta_{c}\right]-\beta\right) u+\operatorname{Im}\left[\beta_{c}\right] \sqrt{\left(u_{2}-u\right)\left(u-u_{1}\right)}$.

This result generalizes Eq. (22) to the case where the convergence point $\beta_{c}$ is complex. Equation (22) is recovered for $\operatorname{Im}\left[\beta_{c}\right]=0$ with an appropriate choice of $\mathcal{M}$.

The large-deviation function can also be written as

$$
\frac{\ln P(u)}{N} \simeq \mathcal{N}-\beta u-\frac{1}{2}\left[\sqrt{\beta_{+}\left(u-u_{2}\right)}-\sqrt{\beta_{-}\left(u-u_{1}\right)}\right]^{2}
$$

for $u_{1} \leqslant u \leqslant u_{2}$, where $\mathcal{N}$ is a normalization constant and we have defined $\beta_{ \pm}=\left|\beta_{c}\right| \pm \operatorname{Re}\left[\beta_{c}\right]$. This result generalizes the expression from Ref. [39] by including a nonzero control parameter, here the inverse temperature $\beta$. Interestingly, the shape of the large-deviation function is given by the upper part of a tilted ellipse [39,60-62]. The tilt is determined by the difference between the real part of the convergence point $\operatorname{Re}\left[\beta_{c}\right]$ and the inverse temperature $\beta$. The width is mainly controlled by the imaginary part $\operatorname{Im}\left[\beta_{c}\right]$. Thus, from the convergence points we can predict the shape and the tilt of the ellipse. The vertical offset $\mathcal{N}$ together with $u_{1}$ and $u_{2}$ must be determined by fitting the ellipse to the exact large-deviation function.

In Fig. 7 we show the large-deviation statistics for three different temperatures. Together with the exact results, we show the ellipse given by Eq. (47) with the convergence points extracted in Fig. 6. We see how the Lee-Yang zeros fully capture the large-deviation statistics with the tilt of the ellipse controlled by the temperature. The fitting parameters $u_{1}, u_{2}$, and $\mathcal{N}$ are only weakly dependent on the temperature. This dependence comes about due to the ansatz in Eq. (44) which only retains the linear dependence on the inverse temperature. The weaker temperature dependence is encoded in the parameters $c_{1}, c_{2}$, and $c_{3}$. It is remarkable how the large-deviation statistics can be related to the Lee-Yang zeros. Moreover, the 
assumption that the saddle points are given by the square-root branch points of the largest eigenvalue of the transfer matrix may be valid in many cases. We thus expect that the relation between Lee-Yang zeros and the large-deviation statistics may hold for many thermodynamic systems with coexisting phases.

\section{FLUCTUATION RELATION}

Finally, we discuss the symmetry properties of the largedeviation statistics [63]. In a spirit similar to that of Ref. [62], it follows easily from Eq. (47) that the fluctuations for large systems obey the symmetry relation

$$
\frac{1}{N} \ln \left[\frac{P(u=\bar{u}+v)}{P(u=\bar{u}-v)}\right]=2\left(\operatorname{Re}\left[\beta_{c}\right]-\beta\right) v
$$

where we have introduced the average $\bar{u}=\left(u_{1}+u_{2}\right) / 2$. This relation resembles the Gallavotti-Cohen fluctuation theorem from nonequilibrium statistical mechanics $[64,65]$. However, rather than the entropy production, this fluctuation symmetry concerns the departure $v$ from the average value $\bar{u}$. The slope on the right-hand side is fully determined by the real part of the convergence point minus the inverse temperature. Equation (49) has a clear physical interpretation. For $\beta=$ $\operatorname{Re}\left[\beta_{c}\right]$, fluctuations away from $\bar{u}$ are equally likely in both directions. Hence, the system has no tendency to settle either to the closed or the open phase. Instead, the microstate of the system is determined by large fluctuations which increase as the Lee-Yang zeros approach the real axis. For $\beta>\operatorname{Re}\left[\beta_{c}\right]$, the probability of fluctuations that involve the opening of links is exponentially suppressed compared to the probability of fluctuations, where links are closed. Thus, the system is forced into the closed state and any departure from it is exponentially unlikely. The analogous argument holds for $\beta<\operatorname{Re}\left[\beta_{c}\right]$. In Fig. 8, we check the expected fluctuation symmetry using the results from Fig. 7 and find good agreement with Eq. (49). This finding is suggestive of an intimate connection between Lee-Yang zeros and fluctuation relations, in both equilibrium and nonequilibrium situations.

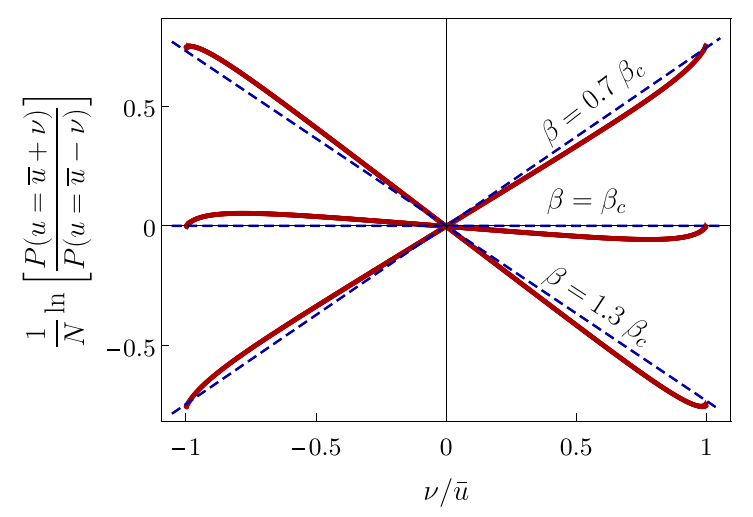

FIG. 8. Fluctuation relation. The solid lines show the left-hand side of Eq. (49) using the exact results from Fig. 7. The dashed lines correspond to the right-hand side of Eq. (49).

\section{CONCLUSION}

We have investigated a thermal phase transition in a molecular zipper using a recently established method to extract the dominant Lee-Yang zeros from fluctuations of thermodynamic observables at finite size. In the present case, we extracted the Lee-Yang zeros in the complex plane of the inverse temperature from the fluctuations of the energy. Other conjugate variables in classical systems would also work. For instance, in a magnetic system, the Lee-Yang zeros in the complex plane of the magnetic field can be extracted from the fluctuations of the magnetization. We have shown that we can predict the temperature at which a phase transition occurs in the thermodynamic limit from the energy fluctuations in small zippers, also at temperatures well above (or below) the transition point. Even if the system does not undergo a sharp transition, the Lee-Yang zeros carry important information about the large-deviation statistics and its symmetry properties. Our work suggests an interesting duality between fluctuations in small systems and their phase behavior in the thermodynamic limit. These predictions may be tested in future experiments on scalable many-body systems. Moreover, the general framework presented here is not restricted to equilibrium systems. It can also be applied to nonconventional phase transitions such as dynamical phase transitions in quantum systems after a quench or dynamic order-disorder transitions in glasses. Adapting these ideas to quantum phase transitions constitutes another interesting line of research.

\section{ACKNOWLEDGMENTS}

We thank J. P. Garrahan and Y. Kafri for insightful discussions. K.B. acknowledges financial support from Academy of Finland (Contract No. 296073). The work was supported by Academy of Finland (Project No. 308515).

\section{APPENDIX A: PRODUCT EXPANSION}

The product expansion of an entire function $f(z)$ around $z=0$ has the general form [46]

$$
f(z)=f(0) e^{z f^{\prime}(0) / f(0)} \prod_{n}\left(1-\frac{z}{z_{n}}\right) e^{z / z_{n}},
$$

where the prime indicates the first derivative with respect to $z$ and the product runs over all complex zeros $z_{n}$ of $f(z)$. For the partition function in Eq. (5), the product expansion explicitly reads

$$
Z=\frac{1-g^{N+1}}{1-g} e^{\beta \varepsilon A_{N}} \prod_{n=-\infty}^{\infty} \prod_{m=1}^{N}\left(1-\frac{\beta \varepsilon}{a_{n m}}\right) e^{\beta \varepsilon / a_{n m}},
$$

where we have introduced

$$
A_{N}=(N+1) \frac{g^{N+1}}{1-g^{N+1}}-\frac{g}{1-g}
$$

and

$$
a_{n m}=\ln g-2 \pi i\left(\frac{m}{N+1}+n\right) .
$$




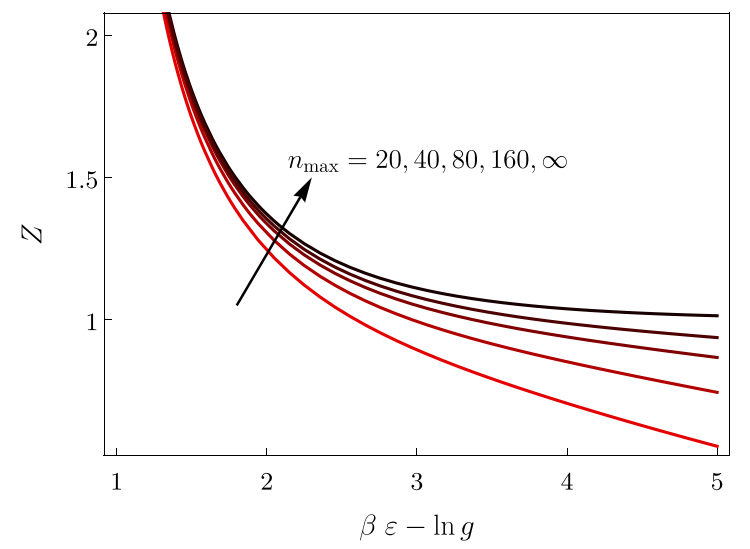

FIG. 9. Product expansion of the partition function. The dashed lines show Eq. (A2) for $N=20$. We have truncated $n$ from $-n_{\max }$ to $+n_{\max }$. With increasing values of $n_{\max }$, the product expansion converges towards the exact partition function for the zipper model shown with a black line.

In Fig. 9 we compare the exact expression for the partition function with the production expansion (A2). The production expansion converges towards the exact result as an increasing number of zeros are included.

\section{APPENDIX B: PROBABILITY DENSITY}

We consider a system with $N$ sites in the canonical ensemble with energies $E_{n}$ and corresponding degeneracy factors $g_{n}$. The partition function can be written as

$$
Z(\beta)=\sum_{n=1}^{\Omega(N)} g_{n} e^{-\beta E_{n}},
$$

where $\beta=1 / k_{B} T$ is the inverse temperature and $\Omega(N)$ is the number of possible energies. For large systems $N \gg 1$, the partition sum can be approximated as

$$
\begin{aligned}
Z(\beta) & =N \sum_{n=1}^{\Omega(N)} \frac{g_{n}}{N} e^{-\beta N\left(E_{n} / N\right)} \\
& \simeq N \int_{0}^{u_{\max }} d u g(u) e^{-\beta N u},
\end{aligned}
$$

where $u_{\max }$ is the maximal energy per site and $g(u)$ is the density of states. Since the Boltzmann factor decays rapidly for large $N$, we can write the partition sum as

$$
Z(\beta) \simeq N \int_{0}^{\infty} d u g(u) e^{-\beta N u}
$$

by extending the upper limit of the integral to infinity.

From the partition sum, we can identify the probability density of finding the system with energy $u$ per site as

$$
P(u)=\frac{N g(u) e^{-\beta N u}}{Z(\beta)} .
$$

It is now convenient to consider the ratio

$$
X(\gamma)=\frac{Z(\beta+\gamma)}{Z(\beta)} \simeq \int_{0}^{\infty} d u P(u) e^{-\gamma N u}
$$

of the partition function at inverse temperatures $\beta+\gamma$ and $\beta$. Specifically, we see that $X(\gamma)$ can be considered as the Laplace transform of the probability density $P(u)[66]$. Thus, through an inverse Laplace transformation, we find

$$
P(u) \simeq \frac{N}{2 \pi i} \int_{c-i \infty}^{c+i \infty} d \gamma X(\gamma) e^{\gamma N u},
$$

where the real constant $c$ has to be chosen such that the integral $\int_{0}^{\infty} d u P(u) e^{-c u}$ is finite. Since $\int_{0}^{\infty} d u P(u)=1$, we are free to set $c=0$. Finally, by a change of variable we find the expression used in the main text,

$$
P(u) \simeq \frac{N}{2 \pi} \int_{-\infty}^{+\infty} d \gamma X(i \gamma) e^{i \gamma N u}
$$

[1] H. B. Callen, Thermodynamics and an Introduction to Thermostatics, 2nd ed. (Wiley, New York, 1985).

[2] D. Chandler, Introduction to Modern Statistical Mechanics, 1st ed. (Oxford University Press, New York, 1987).

[3] N. Goldenfeld, Lectures on Phase Transitions and the Renormalization Group, 1st ed. (Westview, Boulder, 1992).

[4] M. Born and K. Fuchs, The statistical mechanics of condensing systems, Proc. R. Soc. London Ser. A 166, 391 (1938).

[5] J. C. Maxwell, van der Waals on the continuity of the gaseous and liquid states, Nature (London) 10, 477 (1874).

[6] J. E. Mayer, The statistical mechanics of condensing systems. I, J. Chem. Phys. 5, 67 (1937).

[7] J. E. Mayer and P. G. Ackermann, The statistical mechanics of condensing systems. II, J. Chem. Phys. 5, 74 (1937).

[8] J. E. Mayer and E. Montroll, Molecular distribution, J. Chem. Phys. 9, 2 (1941).

[9] C. N. Yang and T. D. Lee, Statistical theory of equations of state and phase transitions. I. Theory of condensation, Phys. Rev. 87, 404 (1952).
[10] T. D. Lee and C. N. Yang, Statistical theory of equations of state and phase transitions. II. Lattice gas and Ising model, Phys. Rev. 87, 410 (1952).

[11] M. E. Fisher, in Lectures in Theoretical Physics, edited by W. E. Brittin (University of Colorado Press, Boulder, 1965), Vol. 7c, p. 1.

[12] S. Grossmann and W. Rosenhauer, Temperature dependence near phase transitions in classical and quant. mech. canonical statistics, Z. Phys. 207, 138 (1967).

[13] S. Grossmann and W. Rosenhauer, Phase transitions and the distribution of temperature zeros of the partition function - I. General relations, Z. Phys. 218, 437 (1969).

[14] S. Grossmann and V. Lehmann, Phase transitions and the distribution of temperature zeros of the partition function - II. Applications and examples, Z. Phys. 218, 449 (1969).

[15] R. A. Blythe and M. R. Evans, The Lee-Yang theory of equilibrium and nonequilibrium phase transitions, Braz. J. Phys. 33, 464 (2003). 
[16] I. Bena, M. Droz, and A. Lipowski, Statistical mechanics of equilibrium and nonequilibrium phase transitions: The YangLee formalism, Int. J. Mod. Phys. B 19, 4269 (2005).

[17] J. Lee, Exact Partition Function Zeros of the Wako-SaitôMuñoz-Eaton Protein Model, Phys. Rev. Lett. 110, 248101 (2013).

[18] J. Lee, Exact partition function zeros of the Wako-Saitô-MuñozEaton $\beta$ hairpin model, Phys. Rev. E 88, 022710 (2013).

[19] P. F. Arndt, S. R. Dahmen, and H. Hinrichsen, Directed percolation, fractal roots and the Lee-Yang theorem, Physica A 295, 128 (2001).

[20] S. M. Dammer, S. R. Dahmen, and H. Hinrichsen, Yang-Lee zeros for a nonequilibrium phase transition, J. Phys. A 35, 4527 (2002).

[21] M. Krasnytska, B. Berche, Y. Holovatch, and R. Kenna, Violation of Lee-Yang circle theorem for Ising phase transitions on complex networks, Europhys. Lett. 111, 60009 (2015).

[22] M. Krasnytska, B. Berche, Y. Holovatch, and R. Kenna, Partition function zeros for the Ising model on complete graphs and on annealed scale-free networks, J. Phys. A 49, 135001 (2016).

[23] P. Borrmann, O. Mulken, and J. Harting, Classification of Phase Transitions in Small Systems, Phys. Rev. Lett. 84, 3511 (2000).

[24] O. Mülken, P. Borrmann, J. Harting, and H. Stamerjohanns, Classification of phase transitions of finite Bose-Einstein condensates in power-law traps by Fisher zeros, Phys. Rev. A 64, 013611 (2001).

[25] W. van Dijk, C. Lobo, A. MacDonald, and R. K. Bhaduri, Fisher zeros of a unitary Bose gas, Can. J. Phys. 93, 830 (2015).

[26] K. P. Gnatenko, A. Kargol, and V. M. Tkachuk, Two-time correlation functions and the Lee-Yang zeros for an interacting Bose gas, Phys. Rev. E 96, 032116 (2017).

[27] R. A. Blythe and M. R. Evans, Lee-Yang Zeros and Phase Transitions in Nonequilibrium Steady States, Phys. Rev. Lett. 89, 080601 (2002).

[28] M. Heyl, A. Polkovnikov, and S. Kehrein, Dynamical Quantum Phase Transitions in the Transverse-Field Ising Model, Phys. Rev. Lett. 110, 135704 (2013).

[29] M. Azimi, M. Sekania, S. K. Mishra, L. Chotorlishvili, Z. Toklikishvili, and J. Berakdar, Pulse and quench induced dynamical phase transition in a chiral multiferroic spin chain, Phys. Rev. B 94, 064423 (2016).

[30] M. Merolle, J. P. Garrahan, and D. Chandler, Spacetime thermodynamics of the glass transition, Proc. Natl. Acad. Sci. USA 102, 10837 (2005).

[31] J. P. Garrahan, R. L. Jack, V. Lecomte, E. Pitard, K. van Duijvendijk, and F. van Wijland, Dynamical First-Order Phase Transition in Kinetically Constrained Models of Glasses, Phys. Rev. Lett. 98, 195702 (2007).

[32] L. O. Hedges, R. L. Jack, J. P. Garrahan, and D. Chandler, Dynamic order-disorder in atomistic models of structural glass formers, Science 323, 1309 (2009).

[33] T. Speck, A. Malins, and C. P. Royall, First-Order Phase Transition in a Model Glass Former: Coupling of Local Structure and Dynamics, Phys. Rev. Lett. 109, 195703 (2012).

[34] C. Binek, Density of Zeros on the Lee-Yang Circle Obtained from Magnetization Data of a Two-Dimensional Ising Ferromagnet, Phys. Rev. Lett. 81, 5644 (1998).

[35] B.-B. Wei and R.-B. Liu, Lee-Yang Zeros and Critical Times in Decoherence of a Probe Spin Coupled to a Bath, Phys. Rev. Lett. 109, 185701 (2012).
[36] X. Peng, H. Zhou, B.-B. Wei, J. Cui, J. Du, and R.-B. Liu, Experimental Observation of Lee-Yang Zeros, Phys. Rev. Lett. 114, 010601 (2015).

[37] J. P. Garrahan and I. Lesanovsky, Thermodynamics of Quantum Jump Trajectories, Phys. Rev. Lett. 104, 160601 (2010).

[38] C. Flindt and J. P. Garrahan, Trajectory Phase Transitions, Lee-Yang Zeros, and High-Order Cumulants in Full Counting Statistics, Phys. Rev. Lett. 110, 050601 (2013).

[39] K. Brandner, V. F. Maisi, J. P. Pekola, J. P. Garrahan, and C. Flindt, Experimental Determination of Dynamical Lee-Yang Zeros, Phys. Rev. Lett. 118, 180601 (2017).

[40] H. Touchette, The large deviation approach to statistical mechanics, Phys. Rep. 478, 1 (2009).

[41] C. Kittel, Phase transition of a molecular zipper, Am. J. Phys. 37, 917 (1969).

[42] D. Poland and H. A. Scheraga, Phase transitions in one dimension and the helix-coil transition in polyamino acids, J. Chem. Phys. 45, 1456 (1966).

[43] Y. Kafri, D. Mukamel, and L. Peliti, Why is the DNA Denaturation Transition First Order? Phys. Rev. Lett. 85, 4988 (2000).

[44] A. Bar, Y. Kafri, and D. Mukamel, Loop Dynamics in DNA Denaturation, Phys. Rev. Lett. 98, 038103 (2007).

[45] S. M. Bhattacharjee, Field Theories in Condensed Matter Physics (Hindustan Book Agency, Gurgaon, 2001), Chap. 2, p. 69.

[46] G. B. Arfken, H. J. Weber, and F. E. Harris, Mathematical Methods for Physicists: A Comprehensive Guide (Academic, New York, 2012).

[47] J. M. Hickey, C. Flindt, and J. P. Garrahan, Trajectory phase transitions and dynamical Lee-Yang zeros of the Glauber-Ising chain, Phys. Rev. E 88, 012119 (2013).

[48] J. M. Hickey, C. Flindt, and J. P. Garrahan, Intermittency and dynamical Lee-Yang zeros of open quantum systems, Phys. Rev. E 90, 062128 (2014).

[49] R. B. Dingle, Asymptotic Expansions: Their Derivation and Interpretation (Academic, New York, 1973).

[50] M. V. Berry, Universal oscillations of high derivatives, Proc. R. Soc. A 461, 1735 (2005).

[51] C. Flindt, T. Novotný, A. Braggio, and A.-P. Jauho, Counting statistics of transport through Coulomb blockade nanostructures: High-order cumulants and non-Markovian effects, Phys. Rev. B 82, 155407 (2010).

[52] C. Flindt, C. Fricke, F. Hohls, T. Novotný, K. Netočný, T. Brandes, and R. J. Haug, Universal oscillations in counting statistics, Proc. Natl. Acad. Sci. USA 106, 10116 (2009).

[53] C. Fricke, F. Hohls, C. Flindt, and R. J. Haug, High cumulants in the counting statistics measured for a quantum dot, Physica E 42, 848 (2010).

[54] C. Fricke, F. Hohls, N. Sethubalasubramanian, L. Fricke, and R. J. Haug, High-order cumulants in the counting statistics of asymmetric quantum dots, Appl. Phys. Lett. 96, 202103 (2010).

[55] D. Kambly, C. Flindt, and M. Büttiker, Factorial cumulants reveal interactions in counting statistics, Phys. Rev. B 83, 075432 (2011).

[56] J. Zamastil and F. Vinette, Determination of singularities of a function from its perturbation expansion, J. Phys. A 38, 4009 (2005). 
[57] T. Karzig and F. von Oppen, Signatures of critical full counting statistics in a quantum-dot chain, Phys. Rev. B 81, 045317 (2010).

[58] J. A. Cuesta and A. Sánchez, General non-existence theorem for phase transitions in one-dimensional systems with short range interactions, and physical examples of such transitions, J. Stat. Phys. 115, 869 (2004).

[59] H. Touchette, R. J. Harris, and J. Tailleur, First-order phase transitions from poles in asymptotic representations of partition functions, Phys. Rev. E 81, 030101 (2010).

[60] A. N. Jordan and E. V. Sukhorukov, Transport Statistics of Bistable Systems, Phys. Rev. Lett. 93, 260604 (2004).

[61] N. Lambert, F. Nori, and C. Flindt, Bistable Photon Emission from a Solid-State Single-Atom Laser, Phys. Rev. Lett. 115, 216803 (2015).
[62] S. Singh, J. T. Peltonen, I. M. Khaymovich, J. V. Koski, C. Flindt, and J. P. Pekola, Distribution of current fluctuations in a bistable conductor, Phys. Rev. B 94, 241407 (2016).

[63] M. Esposito, U. Harbola, and S. Mukamel, Nonequilibrium fluctuations, fluctuation theorems, and counting statistics in quantum systems, Rev. Mod. Phys. 81, 1665 (2009).

[64] G. Gallavotti and E. G. D. Cohen, Dynamical ensembles in stationary states, J. Stat. Phys. 80, 931 (1995).

[65] G. Gallavotti and E. G. D. Cohen, Dynamical Ensembles in Nonequilibrium Statistical Mechanics, Phys. Rev. Lett. 74, 2694 (1995).

[66] F. W. J. Olver, Asymptotics and Special Functions, 1st ed. (Academic, New York, 1974). 